\title{
MAGNETIC FIELD GENERATION BY GALACTIC WINDS
}

\author{
S.J. SPENCER and L.E. CRAM \\ School of Physics, \\ The University of Sydney, \\ N.S.W. 2006, \\ Australia
}

\begin{abstract}
.
A new mechanism is presented for the local amplification and possible global dynamo maintenance of non-axisymmetric large-scale magnetic fields in disk galaxies. Shear in a galactic wind or large-scale flow of ionised gas with components axial and radial to the disk plane may regenerate large-scale magnetic fields.

Numerical results are presented from kinematic mathematical models based on a local (thin disk) approximation and an exact three-dimensional formulation. The one-dimensional thin-disk model illustrates the possibility of exponential amplification and the resulting local axial spatial structure of large-scale galactic magnetic fields. Three-dimensional results support the possibility of global wind dynamo action.
\end{abstract}

\section{Introduction}

Mean-field dynamo theory is commonly used to explain the maintenance of largescale magnetic fields in galactic disks and in some cases halos (for example Parker 1971, 1979; Moffatt 1978; Zeldovich, Ruzmaikin, \& Sokoloff 1983; Fujimoto \& Sawa 1987; Ruzmaikin et al. 1988). Large-scale magnetic field halos extending many kiloparsecs from the central plane of the disk have been observed for some galaxies such as NGC 4631, NGC 1808 and NGC 891 (Hummel, Sancisi, \& Ekers 1984; Hummel et al. 1988; Dahlem 1990; Hummel \& Dahlem 1990; Beck 1991). An alternative approach to the $\alpha \omega$-dynamo in explaining these observations is the possibility of dynamo mechanisms purely due to the inductive effects of three-dimensional, largescale plasma motions (Spencer \& Cram 1992). This approach may be very useful in view of evidence for large-scale axial and radial wind components of motion in some disk galaxies (Lerche \& Schlickeiser 1981; Sukumar \& Velusamy 1985; Hummel et al. 1988; Dahlem 1990). A local model for the kinematic regeneration of magnetic fields may be constructed which incorporates the essential wind effects for indefinite global dynamo activity. It should be realised though, that in accordance with Cowling's anti-dynamo theorem (see Cowling 1981) the growth of magnetic fields due to these inductive processes is restricted to globally non-axisymmetric modes. Wind global dynamo models may therefore help in explaining both thick magnetic disks and halos, and the dominance of non-axisymmetric modes in some galaxies.

\section{Local Model}

\subsection{Formulation/Assumptions}

We begin with the magnetohydrodynamic induction equation for the evolution of a large-scale magnetic field $\mathbf{B}$ in a turbulent plasma

$$
\frac{\partial \mathbf{B}}{\partial t}=\nabla \times(\mathbf{v} \times \mathbf{B})-\nabla \times(\eta \nabla \times \mathbf{B}) .
$$


Here $\mathbf{v}$ is the large-scale velocity field and $\eta$ is the total magnetic diffusivity enhanced by turbulence. The $\alpha$-effect due to a mean helicity of small-scale motions has been ignored for the purposes of the argument.

A local model for the evolution of the magnetic field may be constructed on the basis of a thin disk approximation. The field is assumed to be evolving in a rotating disk such that the radial scale lengths of the plasma and field are far larger than their axial counterparts. In regions distant from the rotation axis the azimuthal dependency of the field is further assumed to be of much less importance than the axial variation. These conditions may be summarised in cylindrical coordinates $(\rho, \phi, z)$ for the magnetic field vector component $B_{i}$ by the expressions

$$
\left(\frac{\partial B_{i}}{\partial \rho}, \frac{B_{i}}{\rho}, \frac{1}{\rho} \frac{\partial B_{i}}{\partial \phi}\right) /\left(\frac{\partial B_{i}}{\partial z}\right) \longrightarrow 0,
$$

and

$$
\left[\frac{1}{\rho} \frac{\partial}{\partial \rho}\left(\rho \frac{\partial B_{i}}{\partial \rho}\right), \frac{1}{\rho^{2}} \frac{\partial^{2} B_{i}}{\partial \phi^{2}}\right] /\left(\frac{\partial^{2} B_{i}}{\partial z^{2}}\right) \longrightarrow 0 .
$$

Equation (??) may be expanded on the basis of the thin disk expressions (??) and (??) to give the following truncated one-dimensional evolution equations for a magnetic field in a plasma with large-scale velocity field $\mathbf{v}=\left(U_{\rho}, U_{\phi}, U_{z}\right)$ and axially dependent magnetic diffusivity

$$
\begin{aligned}
& \frac{\partial B_{\rho}}{\partial t}=\eta \frac{\partial^{2} B_{\rho}}{\partial z^{2}}-\frac{\partial}{\partial z}\left(U_{z} B_{\rho}\right)+\frac{\partial U_{\rho}}{\partial z} B_{z}+\frac{d \eta}{d z} \frac{\partial B_{\rho}}{\partial z} \\
& \frac{\partial B_{\phi}}{\partial t}=\eta \frac{\partial^{2} B_{\phi}}{\partial z^{2}}-\frac{\partial}{\partial z}\left(U_{z} B_{\phi}\right)+\frac{\partial U_{\rho}}{\partial \rho} B_{\phi}+\frac{\partial U_{\phi}}{\partial z} B_{z}+\omega_{\phi} B_{\rho}+\frac{d \eta}{d z} \frac{\partial B_{\phi}}{\partial z}
\end{aligned}
$$

and

$$
\frac{\partial B_{z}}{\partial t}=\eta \frac{\partial^{2} B_{z}}{\partial z^{2}}-\omega_{\rho} B_{z}-U_{z} \frac{\partial B_{z}}{\partial z}+\frac{\partial U_{z}}{\partial \rho} B_{\rho} .
$$

Here the terms

$$
\begin{gathered}
\omega_{\phi}=\rho \frac{\partial}{\partial \rho}\left(\frac{U_{\phi}}{\rho}\right), \\
\omega_{\rho}=\frac{1}{\rho} \frac{\partial}{\partial \rho}\left(\rho U_{\rho}\right)
\end{gathered}
$$

are representative of differential rotation and radial motion. It is assumed that the large scale plasma motions are axisymmetric.

Boundary conditions are imposed on the magnetic field at the galactic midplane and a scale height external to the edge of the current generating region. The mid-plane boundary conditions correspond to an even magnetic mode, while the outer computational boundary is constrained to zero magnetic field. In order to fully define the problem an initial 'seed' magnetic field is also specified throughout the computational domain. 


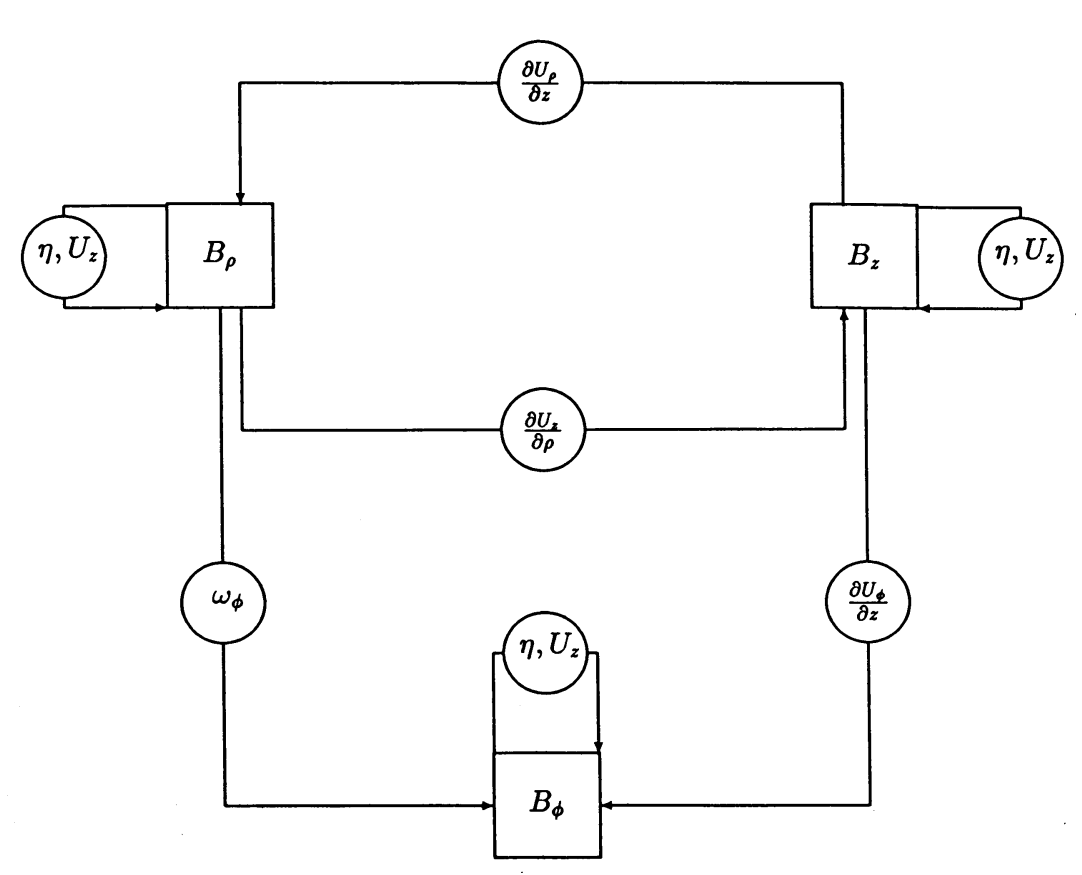

Fig. 1. Schematic of local regeneration model interactive processes. The coupling between the field components in boxes is represented by arrows. Circled terms depict the predominant mechanisms of velocity shear, axial convection and diffusion.

\subsection{Discussion}

Equations (??), (??), and (??) are a set of one-dimensional, time dependent partial differential equations (PDE's) with convective-diffusive and coupling terms. Inductive effects due to shear in the large-scale plasma motions provide the coupling between the magnetic field component evolution equations. It is these coupling terms that are essential to regenerative processes. A fundamental cycle of regeneration of radial and axial components of field is possible, due to shear in both the non-azimuthal components of large-scale plasma motion. Equation (??) shows that the radial component of magnetic field may be induced from the axial component by axial shear in the radial plasma motions. Conversely, the axial component of magnetic field may be induced from the radial component by radial shear in the axial plasma motions. Both the radial and axial components of magnetic field may act as sources for the azimuthal component of field, with induction by shear in the azimuthal component of motion. Diffusive and convective terms may temporarily enhance the field in certain spatial regions, but generally act as global loss mechanisms. Fig. 1 outlines the roles of various dominant terms in magnetic field growth and decay.

Local amplification of magnetic fields may occur for a positive product of the shear in the axial and radial large-scale components of motion. It can also be shown that the strength of this product of mechanisms averaged over the disk thickness must be larger than the product of the characteristic field convective/diffusive loss 
times of the axial and radial components of magnetic field (Spencer \& Cram 1992).

\subsection{Example Results of the local Model}

Solution of the coupled PDE's (??),(??), and (??) has been achieved by a variety of finite difference techniques (for details see Spencer \& Cram 1992). Indefinite local amplification of magnetic fields has been found for a wide variety of large-scale fluid motions representative of both 'open' and 'closed' winds. These are cases of large-scale plasma motions exclusively directed away from the galactic disk, such that they are respectively finite or zero at the outer computational boundary.

Results generally indicate that given suitable large-scale motions, amplification may occur with growth rates of around $10^{9}$ years. An effective magnetic Reynolds number $\left(R_{M}\right)$ may be constructed from characteristic values for the axial component of velocity $\left(U_{z 0}\right)$, total magnetic diffusivity $(\eta)$, and half-thickness $\left(H_{d}\right)$ of the plasma disk $\left(R_{M}=U_{z 0} H_{d} / \eta\right)$. Amplification has been shown for magnetic Reynolds numbers of $R_{M}>200$, although this does not represent a lower bound for effective magnetic Reynolds number with regard to regeneration. It must also be stressed that critical factors in determining the possibility of amplification are characteristic values for the shear in the fluid motions.

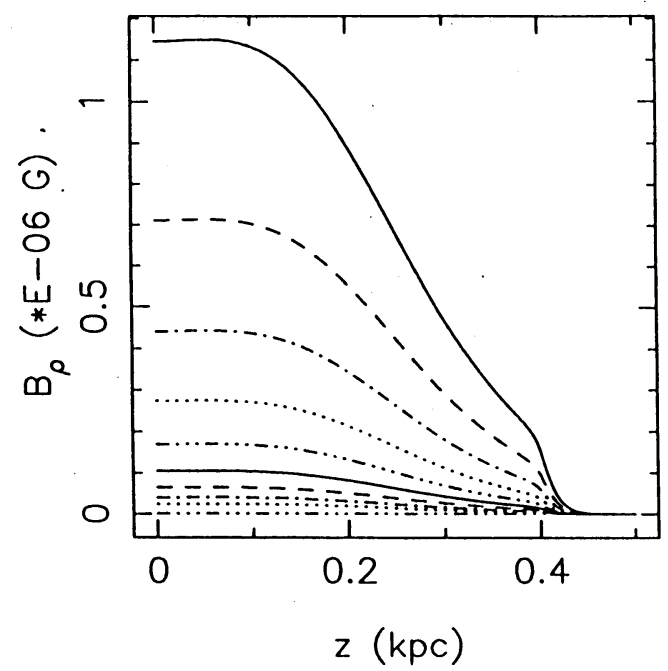

Fig. 2. Evolution of the radial component of magnetic field for an open wind model.

Example results are shown in figs. 2 and 3 for the local amplification of the radial and axial components of a large-scale magnetic field evolving in an 'open' wind. Each line on a magnetic field component evolution figure represents the axial profile at a given time. The profiles are equally spaced in time over a simulation period of $5 \times 10^{9}$ years. This is a sufficient period of time for the dissipation of transient effects due to initial conditions. An initial 'seed' field of $\left(1 \times 10^{-3},-1 \times 10^{-3}, 1 \times 10^{-3}\right) \mu \mathrm{G}$ 
is specified. The wind velocity field is in this case described by

$$
\begin{aligned}
U_{\rho} & =U_{\rho 0}\left(\frac{z}{H_{w}}\right), \\
U_{z} & =U_{z 0}\left(\frac{z}{H_{w}}\right)^{6} .
\end{aligned}
$$

Here the scale height of the wind $H_{w}=0.5 \mathrm{kpc}$ is also the outer computational boundary of the field, while the magnitudes of the wind velocity components are $U_{\rho 0}=75 \mathrm{kms}^{-1}$ and $U_{z 0}=50 \mathrm{kms}^{-1}$. The total magnetic diffusivity is constant at $\eta=0.1 \mathrm{kms}^{-1} \mathrm{kpc}^{-1}$ to scale height of $H_{d}=0.4 \mathrm{kpc}$, beyond which it exponentially increases. The maximal value of the radial shear of the axial component of motion is $30 \mathrm{kms}^{-1} \mathrm{kpc}^{-1}$, the differential rotation is axially constant at $\omega_{\phi}=-26 \mathrm{kms}^{-1} \mathrm{kpc}^{-1}$, and the radial shear in the radial motion $\left(\omega_{\rho}\right)$ is held at zero.

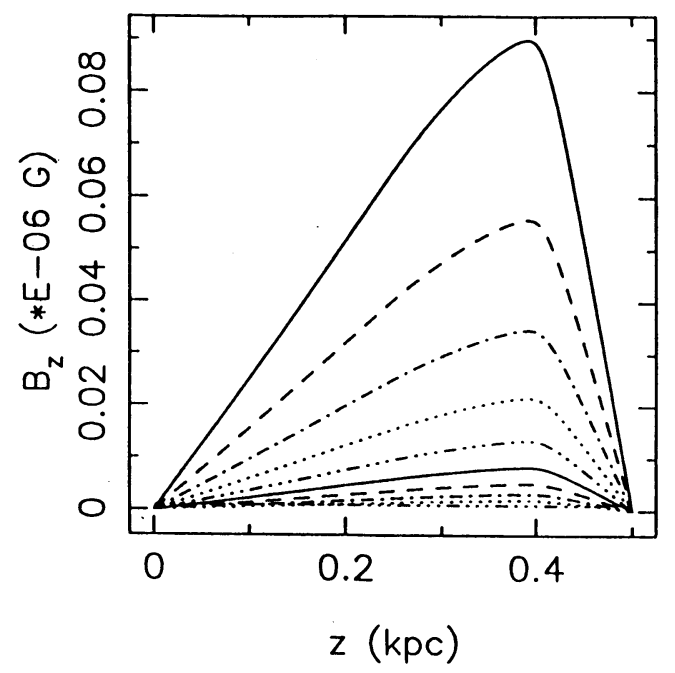

Fig. 3. Evolution of the axial component of magnetic field for an open wind model.

These results show local amplification of the magnetic field. The radial and azimuthal components of field are concentrated near the mid-plane. Spatial distribution of the radial and azimuthal components of field are similar but of reversed sign due to the negative differential rotation. Magnetic field strength is dominated by the azimuthal component such that $\left|B_{\phi} / B_{\rho}\right| \approx 10$. The strength of the axial component is relatively small with $\left|B_{z} / B_{\rho}\right|<0.45$ throughout the computational domain. In contrast to the radial and azimuthal magnetic field, the axial component is concentrated near the diffusive scale height. The axial magnetic field component in regions of relatively small magnetic Reynolds number may therefore counterdiffuse towards the galactic mid-plane.

Magnetic field strength declines in the outer region of large magnetic diffusivity. The increasing total diffusivity in this region is intended to simulate the transition through a halo to a vacuum where the field is current-free. As fig. 2 shows, an 
external region of very high magnetic diffusivity acts to effectively exclude the radial and azimuthal components of magnetic field. The axial magnetic field is also significantly decreased in this region (fig. 3), though not as rapidly as the other two vector components.

\section{Three-Dimensional Global Model}

\subsection{Formulation/Numerical Issues}

It should be realised that results from local models do not provide conclusive evidence of indefinite global dynamo action. Rather, these results provide evidence of local magnetic field amplification. In particular, the global constraint of nonaxisymmetric dynamo maintained fields embodied in Cowling's anti-dynamo theorem must be reflected in the results of a global wind dynamo model. Convective/diffusive losses of magnetic flux in the radial direction must be properly quantified in a fully three-dimensional model. The importance of strong axial gradients of the radial component of motion also implies spatial regions of strong radial advection of magnetic field. This may imply the addition of significant advective loss terms which restrict the range of parameters allowing dynamo activity.

The possibility of global dynamo action for exclusively outward directed winds requires the presence of strong diffusion. Magnetic fields must be able to diffuse towards the galactic body in order for the growth of magnetic field to be indefinite. The case of zero diffusivity is simply amplification by shear of an initial magnetic field while propagating out of the galactic system. This may imply an effective upper bound on the global minimum magnetic Reynolds number for the occurrence of indefinite dynamo action. Magnetic diffusion enhanced by turbulence therefore plays a dual role in the wind dynamo model of both destroying and redistributing magnetic flux. Such a role for magnetic diffusion was envisaged by Cowling (1981) in regard to the maintenance of dynamo activity in the Solar convective cells. A global wind dynamo model must demonstrate the possibility of magnetic counter-diffusion in both the axial and radial directions for outward-directed galactic winds.

A three-dimensional kinematic wind dynamo model has been constructed in cylindrical geometry. As in the local model, the large-scale velocity field is restricted to axisymmetric, three-dimensional plasma motions. Induction of magnetic field by shear in the fluid motions is by the same mechanisms as those presented in the local model. Simulation of the three-dimensional convective/diffusive transport of magnetic field allows the study of wind dynamos in galaxies with thick disks and/or massive halos.

The large-scale magnetic field is written as a superposition of be a sinusoidal functions of azimuthal position

$$
\mathbf{B}(\rho, \phi, z ; t)=\Sigma_{m=0}^{\infty}\left[\mathbf{B}_{m R}(\rho, z)+i \mathbf{B}_{m I}(\rho, z)\right] \exp (i m \phi) .
$$

The assumed axisymmetric form of the velocity field (and all other plasma parameters) allows orthogonality conditions to be used to separate the evolution of individual azimuthal magnetic eigenmodes. 
Three-dimensional evolution equations may be written for the real and imaginary parts of the vector components of magnetic fields in terms of the convection/diffusion and shearing terms. The divergence-free condition on the magnetic field is trivially analytically satisfied over all time for initially divergence-free fields. Unfortunately, numerical methods applied to the evolution equations for the magnetic induction in this form generally do not adequately preserve the divergence-free condition over large integration times. For simulations of $10^{8}$ or more years this generally results in the catastrophic growth of magnetic monopoles. The solution may become so degraded as to be completely unreliable.

Several approaches may be used to numerically satisfy the divergence-free constraint on the evolving magnetic field. The spatial discretisation approach of Elstner, Meinel, \& Rüdiger (1990), which numerically constrains temporal changes of the total EMF and magnetic induction to be divergence-free, has been implemented. The method is only suitable though for relatively low effective magnetic Reynolds numbers $\left(R_{m}<500\right)$, and exhibits numerical instability for more strongly convection dominated problems. This is because the differencing scheme is basically spatially centred and therefore unsuitable for the explicit evolution of transport terms. It should be noted that the constrained transport method of Evans \& Hawley (1988) with suitable choice of spatially differencing scheme for transport terms is undoubtedly more robust and versatile for convection dominated problems. The first order accurate in time, explicit Eulerian scheme and the fourth order accurate in time, explicit Runge-Kutta scheme have both been successfully used for the temporal discretisation.

Both zero magnetic field and impenetrable wall boundary conditions have been used at distant radial and axial computational boundaries. Instabilities may propagate throughout the computational domain if convection is occurring in the distant boundary regions. Open winds may prove to be particularly difficult to simulate given the problems associated with stably implementing appropriate boundary conditions. Despite these constraints, the method of Elstner et al. (1990) is still useful for many wind dynamo problems given adherence to constraints imposed by the chosen time-stepping algorithm, and the localisation of the radial and axial velocity field to regions distant from the outer computational boundaries.

\subsection{Preliminary Results}

Results of the three-dimensional wind dynamo model are preliminary but suggestive in many regards. Simulations of magnetic field evolution in thick disks over $5 \times 10^{8}$ years have shown that BSS fields may globally exponentially grow for appropriate combinations of shearing winds and differential rotation. The total magnetic energy of axisymmetric fields subject to the same conditions initially grows over more than $10^{8}$ years, but in accordance with Cowling's theorem eventually decays under diffusive action because there is no source of poloidal field. Non-axisymmetric magnetic fields also decay if subject to simple diffusive action in a non-rotating or uniformly rotating disk. These same fields initially grow if the rotation is non-uniform, though this action eventually ceases due to diffusive effects. The global exponential growth of magnetic fields due to wind motions is consistent with dynamo action but results 
must be confirmed with longer time integrations (ie $10^{10}$ years as compared to the current $10^{8}-10^{9}$ years). Detailed results from the three-dimensional model will be presented elsewhere.

\section{Conclusions}

Amplification of large-scale magnetic fields may occur due to shearing wind motions. The local model dernonstrates the essence of the regeneration processes, while results from the three-dimensional model are consistent with global dynamo action.

Evolution of the radial and axial components of magnetic field are coupled by axisymmetric shear in the wind motions. Azimuthal magnetic field may be induced from the other field components by shear in the large-scale azimuthal velocity. Large-scale magnetic fields may be amplified if regenerative inductive effects due to shear in the large-scale motions dominate advective and diffusive losses. Global dynamo action may be maintained in a galactic disk for exclusively outward directed winds if the magnetic field can counter-diffuse towards the disk. Enhanced diffusion due to turbulent mixing is of sufficient magnitude for significant regenerative growth of large-scale magnetic fields on galactic time scales.

\section{Acknowledgements}

SJS thanks the IAU, Science Foundation for Physics, and CSIRO Division of Mathematics and Statistics for conference financial support. This work is supported by the Australian Research Council and the Science Foundation for Physics. SJS gratefully acknowledges the financial support of a University of Sydney Postgraduate Research Award.

\section{References}

Beck, R.: 1991, in 'The Interstellar Disk-Halo Connection in Galaxies', IAU Symposium 144, ed. J.B.G.M. Bloemen (Dordrecht:Kluwer), 267.

Cowling, T.G.: 1981, ARA\&A $19,115$.

Dahlem, M.: 1990, 'Disk-halo Interactions in the Spiral Galaxies NGC 1808 and NGC 891', PhD. Thesis (Bonn: Rheinischen Friedrich-Wilhelms Universität)

Ekers, R.D. \& Sancisi, R.: 1977, A\&A 54,973.

Elstner, D., Meinel, R. \& Rüdiger, G.: 1990, GeoApFlDyn 50,85.

Evans, C.R. \& Hawley, J.F.: 1988, ApJ 332,659.

Fujimoto, M. \& Sawa, T.: 1987, Pub.Astr.Soc.Japan 39, 375.

Hummel, E., Lesch, H., Wielebinski, R. \& Schlickeiser, R.: 1988, A\&A 197,L29.

Hummel, E., Sancisi, R. \& Ekers, R.D.: 1984, AGA 133, 1.

Hummel, E. \& Dahlem, M.: 1990, in 'Galactic and Intergalactic Magnetic Fields', IAU Symposium 140, eds. R. Beck, P.P. Kronberg, R. Wielebinski (Dordrecht:Kluwer),219.

Lerche, I. \& Schlickeiser, R.: 1981, Ap. Letters $22,31$.

Moffatt, H.K.: 1978, 'Magnetic Field Generation in Electrically Conducting Fluids' (Cambridge:Cambridge University)

Parker, E.N.: 1971, ApJ 163,255.

Parker, E.N.: 1979, 'Cosmical Magnetic Fields' (Oxford:Clarendon)

Ruzmaikin, A.A., Shukurov, A.M. \& Sokoloff D.D.: 1988, 'Magnetic Fields in Spiral Galaxies' (Dordrecht:Kluwer)

Sofue, Y., Fujimoto, M. \& Wielebinski, R.: 1986, ARA $8 A$ 24,459.

Spencer, S.J. \& Cram, L.E.: 1992, ApJ 400, in press. 
Sukumar, S. \& Velusamy, T.: 1985, MNRAS 212,367.

Zeldovich, Y.B., Ruzmaikin, A.A. \& Sokoloff, D.D.: 1983, 'Magnetic Fields in Astrophysics' (New York:Gordon and Breach) 